\title{
Effects of an Algorithm with a Recommendation Tree for Indirect Speech Acts
}

\author{
Takuki Ogawa, Kazuhiro Morita, Masao Fuketa, and Jun-Ichi Aoe
}

\begin{abstract}
For context-based recommendation systems, it is important to determine intentions from indirect speech acts.

An algorithm of deriving intentions from indirect speech acts has been proposed, but the algorithm included unclear portions and there were no important experimental results for kinds of speech acts. Therefore, this paper proposes an improved algorithm and two experimental observations are discussed for accuracies and kinds of answers in indirect speech acts.

Logical formulas are rewritten to if-else statements and the number of conditions is reduced from 24 to 8 in the algorithm. From experimental results, it is verified that the correct rate of the proposed method is $\mathbf{4 8 . 2}$ points higher than the one of the traditional method in indirect speech acts. Answers of "what" most include indirect speech acts and the accuracy of the proposed method is $\mathbf{5 3 . 8}$ points higher than the traditional one in them.
\end{abstract}

Index Terms-Recommendation system, indirect speech acts, affirmative intention, negative intention.

\section{INTRODUCTION}

Context-based recommendation systems [1]-[9] support users to take items such as products, services, and information from a large choice of them by dialogues. In order to decide recommendation items, affirmative and negative intentions in answers are important for these systems [6]-[9].

In kinds of expressions of these intentions, there are direct speech acts and indirect speech acts. The direct speech acts represent these intentions by the following two approaches for a recommendation "How about having a cake today?": the first is fixed phrases such as "Yes, I have." and "No, thank you." without "cakes". The second is sentences representing acceptance and rejection intentions such as "I like cakes." and "I don't want to have cakes." with "cakes", respectively.

In the indirect speech acts, there are two patterns for the recommendation: the first is the affirmative answers that select other cakes excluding chocolate cakes such as "I don't want to have chocolate cakes.". The second is the negative answers that select other foods excluding cakes such as "I want to have Japanese noodles".

In order to determine intentions from sentences, there are two methods: the first uses machine learning [10]-[16] the

Manuscript received September 24, 2013; revised November 6, 2013

The authors are with University of Tokushima, Tokushima, Japan (email: ogawa-takuki@iss.tokushima-u.ac.jp, kam@is.tokushima-u.ac.jp, fuketa@is.tokushima-u.ac.jp, aoe@is.tokushima-u.ac.jp). second uses meaning of words and grammars [17]-[19]. Methods using machine learning have the advantage that classification models are constructed automatically, but they expend considerable efforts to collect a large learning data. Methods using meaning of words and grammars have the merit that their rules have broad utilities for sentences of many domains, but they can not classify answers of indirect speech acts.

Previously, we defined a recommendation tree and proposed an algorithm of deriving intentions for indirect speech acts [20]. From experimental results, it was verified that the algorithm is superior to the traditional one.

However, the algorithm is very complex and there are no important experimental results for kinds of speech acts.

Therefore, this paper proposes an improved algorithm and two experimental observations are discussed for accuracies and kinds of answers in each speech act.

\section{A ReCOMmendation TREe AND AN ALGORIthm}

First of all, this paper defines recommendation conditions (RC) and recommendations. In RCs, there are three kinds; R_RC, S_RC, and NS_RC. R_RC is the required RC. In a recommendation, "How about having a cake today?", there are R_RCs, "you", "have", "cake", and "today". S_RC is the selectable RC. In the recommendation, there are S_RCs of kinds of cakes such as "chocolate cake", "short cake", and "Mont Blanc". NS_RC is the non-selectable RC such as "tomorrow", "Japanese noodle" for the recommendation. Recommendations are constructed by four necessary concepts of RC: "WHO", "WHEN", "WHAT", and "VERB". These concepts have RCs related to persons such as "you" and "he", schedules such as "today" and "tomorrow", objects such as "cake" and "curry", and actions such as "go" and "have", respectively.

A recommendation tree has root node "REC" which indicates the recommendation. The root node has four child nodes corresponding to concepts (concept nodes): "WHO", "WHEN", "WHAT", and "VERB". These nodes have child nodes of RCs (RC nodes). For example, concept node "WHO" has RC nodes "you" and "he". There are three kinds of edges (R_edges, S_edges, and NS_edges) for R_RC, S_RC, and NS_RC, respectively. The root node and concept nodes are connected by R_edges. Concept nodes and RC nodes are connected by S_edges. These kinds of edges are changed by each recommendation. For the recommendation "How about having a cake today?", edges of nodes "you", "today", "cake", and "have" are modified to R_edges and other edges of nodes are set to NS_edges as shown in Fig. 1. In Fig. 1, the node labeled by $\mathrm{x}$ corresponding to string $\mathrm{x}$. 




Fig. 1. A part of the recommendation tree of "How about having a cake today?"

The recommendation tree can be extended by expanding terminal nodes. Considering an example in Fig. 1, RC node "cake" constructs the subtree as the root node. RC node "cake" has RC nodes "taste" and "kind" with R_edges as child nodes. RC node "taste" has RC node "sweet" with a R_edge as a child node. RC node "kind" has RC nodes "Mont Blanc", "short cake", and "chocolate cake" with S edges as child nodes.

An algorithm determines intentions of answers to derive the recommendation tree [20].

The algorithm is modified more compact and logical formulas are rewritten to if-else statements in this study. Before proposing the algorithm, the following definitions are prepared.

\section{Definition}

Suppose that NODE[x] is a node for string $\mathrm{x}$. PARENT(NODE[x]) represents the parent node of NODE[x]. AS_SIBLING(NODE[x]) returns the set of sibling nodes of NODE[x] with S_edges. Let EDGE[NODE[x],NODE[y]] be the kind of edges (R-edge, S_edge, and NS_edge) between NODE[x] and NODE[y]. Let P_EDGE[NODE[x]] be EDGE[NODE[x],PARENT(NODE[x])]. Let INTENTION[NODE[x]] be the intention of NODE[x] which has one of three kinds intentions: acceptance, rejection, and no_information in this algorithm. No_information means that a node doesn't have any intentions. All intentions of nodes are initialized to no_information.

An algorithm of deriving intentions

\section{Input: ANSWER_NODE[]} and

\section{ANSWER_INTENTION[]}

ANSWER_NODE[] is a list of strings for nodes accepted or rejected by answers. ANSWER_INTENTION[] is a list of intentions for elements in ANSWER_NODE[]. Indexes of ANSWER_INTENTION[] are elements in ANSWER_NODE[]. For the answer "I like curries", ANSWER_NODE[] is $\{$ "curry"\} and ANSWER_INTENTION[“curry"] is \{“acceptance"\}, respectively.

\section{Output: INTENTION[NODE[“REC"]] \\ Method:}

for $i=1$ to $n$ do/*n is the number of elements in ANSWER_NODE[] */

INTENTION[NODE[ANSWER_NODE[i]]= ANSWER_INTENTION[ANSWER_NODE[i]]

target_node $=$ NODE[ANSWER_NODE[i]] while target_node NODE["REC"] do if $\mathrm{P} \_$EDGE[NODE[target_node]] is R_edge then INTENTION[PARENT(NODE[target_node])]

INTENTION[NODE[target_node]] else if P_EDGE[NODE[target_node]] is S_edge then if INTENTION[AS_SIBLING[NODE(target_node)]] = rejection then

INTENTION[PARENT(NODE[target_node] $)=$ rejection else

INTENTION[PARENT(NODE[target_node]) = acceptance

end

else if P_EDGE[NODE[target_node]] is NS_edge then if INTENTION[NODE[x]] = acceptance then INTENTION[PARENT(NODE[x])] = rejection end

end

endwhile

if INTENTION[ NODE["REC"]] is rejection then INTENTION[ NODE[“REC"]] = negative

break

else if INTENTION[ NODE["REC"]] is acceptance then INTENTION [ NODE["REC"]] = affirmative endif

endfor

End of Algorithm

To compare with the previous algorithm, the total number of conditions in logical functions and the algorithm are reduced from 24 to 8 .

In case of a recommendation "How about having a cake today?", examples of derivations from answers "I like something sweet." and "I dislike chocolate cakes" are as follows:

\section{Example 3.1}

For an answer "I like something sweet.", INTENTION[NODE["sweet"]] is acceptance. From Fig. 1., P_EDGE[NODE["sweet"]] is R_edge. Therefore, INTENTION[NODE["taste"]] is acceptance. Similarly, intentions of NODE["cake"], NODE["WHAT"], NODE["REC"] are acceptance.

From these results, the intention of the answer is affirmative.

\section{Example 3.2}

For an answer "I dislike chocolate cakes", INTENTION[NODE["chocolate cake"]] is rejection. From Fig. 1., P_EDGE[NODE["chocolate cake"]] is S_edge. AS SIBLING(NODE[“chocolate cake"]) isn't rejection. Therefore, INTENTION[NODE["kind"]] is acceptance. 
INTENTION[NODE["kind"]] is acceptance because P_EDGE[NODE["kind"]] is R_edge. Similarly, NODE["cake"], NODE["WHAT"], NODE["REC"] are acceptance.

From these results, the intention of the answer is affirmative.

\section{EXPERIMENTS AND DISSCUSSIONS}

\section{A. Knowledge for Experiments}

It is general to recommend foods including cakes and Japanese noodles, and movies on a daily basis. In this experiment, the following three recommendations are assumed, where "Resident Evil" is a title of a movie:

Recommendation "cake": "How about having a cake today?"

Recommendation "Japanese noodle": "How about having a Japanese noodle today?"

Recommendation "Resident Evil": "How about going to the movie, Resident Evil?"

In order to determine intentions from answers to them, a recommendation tree is constructed from closed corpora which have 500 answers for each recommendation. Answers are collected by four undergraduate students. From corpora, $\mathrm{RC}$ nodes and the recommendation tree are defined by discussions with these students. Total numbers of nodes are 311. Examples of RC nodes with concept nodes are presented in Table I.

For RC nodes "cake", "Japanese noodle", and "Resident Evil", more detailed descendant nodes are constructed. Examples of descendant nodes for each RC node are presented in Table II. In Table II, R_edge and S_edge between a node and a parent node show $\mathrm{R}$ and $\mathrm{S}$, respectively.

TABLE I: EXAMPLES OF RC NODES WITH CONCEPT NODES

\begin{tabular}{|l|l|}
\hline Concept nodes & RC nodes \\
\hline WHO & Father, Mother, You, She \\
\hline WHEN & Morning, Today, this week \\
\hline WHAT & Cake, Japanese noodle, Resident Evil \\
\hline VERB & Eat, need, watch \\
\hline
\end{tabular}

TABLE II: EXAMPLES OF DESCENDANT NODES OF NODES “CAKE”, "JAPANESE NOODLE", AND "RESIDENT EVIL"

\begin{tabular}{|l|ll|}
\hline Parent & Child & Grandchild \\
\hline Cake & Genre[R] & Sweets[R],Dessert[R] \\
& Taste[R] & Sweet[R] \\
& Kind[R] & Short cake[S], chocolate cake[S] \\
& Ingredient[R] & Flour[R], Sugar[R] \\
& & Butter[S], Apple[S] \\
& Genre[R] & Noodles[R], Food[R] \\
Japanese & Taste[R] & Spicy[S], Salty[S] \\
noodle & Ingredient[R] & Flour[R] \\
& Screen type[R] & Garlic[S], Bean sprouts[S] \\
\hline Resident & Caption[S], Dub[S], 3D[S] \\
Evil & Genre of films[R] & Horror[R], Action[R] \\
\hline
\end{tabular}

( $\mathrm{R}$ and $\mathrm{S}$ means required and selectable)

\section{B. Experimental Results}

\section{1) Experimental results for each recommendation}

In order to evaluate the accuracy of the proposed method, open tests are carried out. Open tests uses corpora with 120 answers for each recommendation. These corpora are collected by eleven students who don't accumulate closed corpora, and they make answers to each recommendation without restriction of responses. The traditional method proposed by Yoshie et al. [19] is used as a comparative method. Fig. 2 shows correct rates for each recommendation of the proposed method and the comparative method, respectively. Correct rates mean percentages of correctly classified sentences in total sentences.

In Fig. 2., all accuracies of the proposed method are about 40 points higher than the comparative method in open tests of all recommendations.

From these results, it is verified that the proposed method is much better than the traditional method to determine intentions.

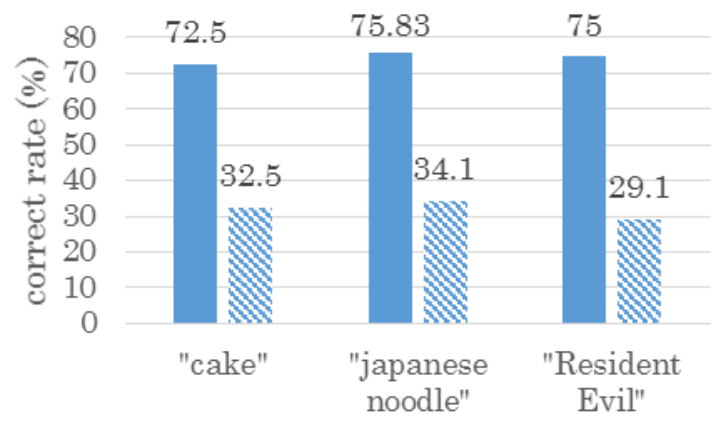

Recommendations

- proposed method $\mathbb{N}$ comparative method

Fig. 2. Correct rates for each recommendation.

TABLE III: CORRECT RATES OF EACH SPEECH ACT

\begin{tabular}{|l|r|r|}
\hline & Proposed method (\%) & Traditional method (\%) \\
\hline Direct speech acts & 85.8 & 54.4 \\
\hline $\begin{array}{l}\text { Indirect speech } \\
\text { acts }\end{array}$ & 68.2 & 20 \\
\hline
\end{tabular}

\section{2) Correct rates of each speech act}

From experimental results, accuracies of methods in direct speech acts and indirect speech acts are found out. Table III shows correct rates of each speech act.

From Table III, accuracies of the proposed method in speech acts are higher than the traditional method. Especially the difference in correct rates of indirect speech acts is 48.2 points.

This result shows that the proposed method is more effective than the traditional method to determine intentions from indirect speech acts.

\section{3) Rates of indirect speech acts}

In order to bring out kinds of answers which often include indirect speech acts, 360 answers of open tests are classified into kinds of their targets of intentions, recommendation, who, when, what, and V. Table IV shows that rates of indirect speech acts per targets of answers. 
Table IV shows that indirect speech acts are often included in answers of "when" and "what". In these answers, correct rates of the proposed method and the traditional method are compared. Table V shows correct rates of methods in these answers.

From Table V, correct rates of the proposed method are 37.7 points and 53.8 points higher than the traditional method in answers "when" and "what", respectively. To compare kinds of answers, the correct rate of "what" is 39.2 points higher than the one of "when" in the proposed method. The reason of this result is that concept node "what" has more detailed child nodes than node "when".

From these results, it is verified that the proposed method with the detailed recommendation tree is more effective to determine intentions from indirect speech acts than the traditional one.

TABLE IV: RATES OF INDIRECT SPEECH ACTS PER TARGETS OF ANSWERS

\begin{tabular}{|l|l|}
\hline & Rates of indirect speech acts $(\%)$ \\
\hline Recommendation & 0.83 \\
\hline Who & 1.94 \\
\hline When & 25.0 \\
\hline What & 36.7 \\
\hline V & 0.83 \\
\hline
\end{tabular}

TABLE V: CORRECT RATES OF METHODS IN ANSWERS OF “WHEN" AND "WHAT"

\begin{tabular}{|l|l|l|}
\hline & $\begin{array}{l}\text { Proposed method } \\
(\%)\end{array}$ & $\begin{array}{l}\text { Traditional method } \\
(\%)\end{array}$ \\
\hline When & 43.3 & 5.6 \\
\hline What & 82.5 & 28.7 \\
\hline
\end{tabular}

\section{CONCLUSIONS}

This paper has proposed an improved algorithm, and two experimental observations have been discussed for accuracies and kinds of answers in each speech act.

Logical formulas are rewritten to if-else statements and the number of conditions is reduced from 24 to 8 in the algorithm. From experimental results, it is verified that the correct rate of the proposed method is 48.2 points higher than the one of the traditional method in indirect speech acts. Answers of "what" most include indirect speech acts and the accuracy of the proposed method is 53.8 points higher than the traditional one in them.

\section{REFERENCES}

[1] A. Levi, O. Monkryn, C. Diot, and N. Traft, "Finding a needle in a haystack of reviews: cold start context-based hotel recommender system demo," in Proc. 6th ACM Conference on Recommender Systems, New York, 2012, pp. 115-122.

[2] P. Lops, G. Marco, and S. Giovanni, "Content-based recommender systems: State of the art and trends," in Recommender Systems Handbook, Springer US, 2011, pp. 73-105.

[3] B. J. Han, S. Rho, S. Jun, and E. Hwang, "Music emotion classification and context-based music recommendation," Multimedia Tools and Applications, vol. 47, no. 3, pp. 433-460, 2010.

[4] P. Johansson, "Natural language interaction in personalized EPGs," in Proc. 3rd International Workshop on Personalization of Future TV, Johnstown Pennsylvania, 2003, pp. 27-31.
[5] H. Shimazu, "ExpertClerk: navigating shoppers' buying process with the combination of asking and proposing," in Proc. 17th IJCAI, 2001, pp. 1443-1448.

[6] P. Johansson, "Madfilm-a multimodal approach to handle search and organization in a movie recommendation system," in Proc. 1st Nordic Symposium on Multimodal Communication, 2003, pp. 53-65.

[7] T. Misu and T. Kawahara, "Speech-based interactive information guidance system using question-answering technique," in Proc. ICASSP 2007 Conf., 2007, pp. 145-148.

[8] H. Shimazu, "ExpertClerk: A Conversational Case-Based Reasoning Tool forDeveloping Salesclerk Agents in E-Commerce Webshops," Artificial Intelligence Review, vol. 18, issue 3-4, pp. 223-244, 2002.

[9] C. A. Thompson, M. H. Goeker, and P. Langley, "A personalized system for conversational recommendations," Journal of Artificial Intelligence Research, vol. 21, issue 1, pp. 393-428, January 2004.

[10] R. Fernandez and R. W. Picard, "Dialog act classification from prosodic features using support vector machines," in Proc. Speech Prosody 2002 Conf., 2002, pp. 291-294.

[11] D. Surendran and G. A. Levow, "Dialog act tagging with support vector machines and hidden Markov models," in Proc. Interspeech, 2006, pp. 1950-1953.

[12] A. Stolcke, K. Ries, N. Coccaro, E. Shriberg, R. Bates, D. Jurafsky, P. Taylor, R. Martin, C. V. Ess-Dykema, and M. Meteer, "Dialogue act modeling for automatic tagging and recognition of conversational speech," Computational Linguistics, vol. 26, issue 3, pp. 339-373, September 2000.

[13] S. Ravi and J. Kim, "Profiling student interactions in threaded discussions with speech act classifiers," in Proc. 2007 Artificial Intelligence in Education Conf., 2007, pp. 357-364.

[14] K. Mera, T. Ichimura, and T. Yamashita, "Analysis of User Communicative Intention from Affirmative/Negative Elements by Fuzzy Reasoning and Its Application to WWW-based Health Service System for Elderly," in Proc. 6th Soft Computing Conf., IIZUKA Japan, 2000, pp. 971-976.

[15] M. Louwerse and S. Crossley, "Dialog Act Classification Using N-Gram Algorithms," in Proc. FLAIRS Conf., Menlo Park, 2006, pp. 758-763.

[16] N. Webb, M. Hepple, and Y. Wilks, "Dialogue act classification based on intra-utterance features," in Proc. AAAI Workshop on Spoken Language Understanding, Pittsburgh, 2005.

[17] J. Kitamura, Y. Watanabe, Y. Sekiguchi, and Y. Suzuki, "An Extraction and Processing Method of User's Denial Utterance for a Speech Dialog Device," Trans. of Information Processing Society of Japan, vol. 46, no. 7, pp. 1789-1796, July 2005.

[18] K. Mera, "Analyzing affirmative/negative intention from plural sentences," in Proc. KES'01, Osaka/Nara, 2001, pp. 1222-1227.

[19] M. Yoshie, K. Mera, T. Ichimura, T. Yamashita, T. Aizawa, and K. Yoshida, "Analysis of affirmative/negative intentions of the answers to yes-no questions and its application to a web-based interface," Journal of Japan Society for Fuzzy Theory and Systems, vol. 14, no. 4, pp. 393-403, August 2002.

[20] T. Ogawa, K. Morita, M. Fuketa, and J. Aoe, "The Determination of Affirmative and Negative Intentions for Indirect Speech Acts by a Recommendation Tree," International Journal of Advanced Computer Science and Applications, vol. 4, issue 8, pp. 228-235, August 2013.

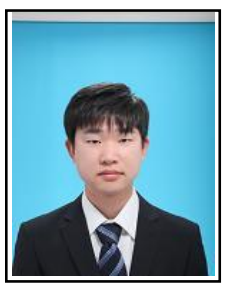

Takuki Ogawa received his $\mathrm{BSc}$ and $\mathrm{MCsD}$ in Information Science and Intelligent System from University of Tokushima, Japan, in 2010 and 2012. He is a Ph.D student at University of Tokushima. His main research interests are natural language processing, dialog systems and communication robots.

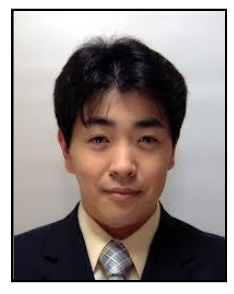

Kazuhiro Morita received B.Sc., M.Sc. and Ph.D Degrees in information science and Intelligent systems from University of Tokushima, Japan in 1995, 1997 and 2000. Since 2000, he has been a research assistant of the Department of the Department of Information Science \& Intelligent Systems, Tokushima University, Japan. His research interests in Sentence retrieval from huge text data bases, double-array structure and Binary search tree. 


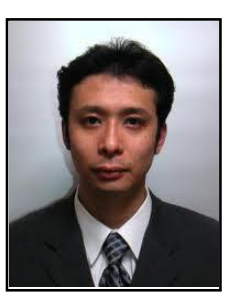

Masao Fuketa received B.Sc., M.Sc. and Ph.D. Degrees in information science and Intelligent systems from University of Tokushima, Japan, in 1993, 1995 and 1998. He has been a research assistant from 1998 to 2000 in information science and Intelligent Systems from University of Tokushima, Japan. He is currently a research associate in the department of Information Science \& Intelligent Systems, Tokushima University, Japan. He is a member in the Information processing Society in Japan and The Association for Natural language processing of Japan. His research interests in Sentence retrieval from huge text data bases and morphological analysis.

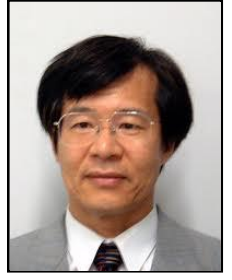

Jun-Ichi Aoe received B.Sc. and M.Sc. Degrees in electronic engineering from the University of Tokushima, Japan, in 1974 and 1976, respectively, and the $\mathrm{Ph} . \mathrm{D}$. degree in communication engineering from the University of Osaka, Japan 1980. Since 1976 he has been with the University of Tokushima. He is currently a professor in the department of Information Science \& Intelligent Systems, Tokushima University, Japan. His research interests in natural language processing, a shift-search strategy for interleaved LR parsing, robust method for understanding $\mathrm{NL}$ interface commands in an intelligent command interpreter, and trie compaction algorithms for large key sets. He was the editor of the computer Algorithm Series of the IEEE computer Society Press. $\mathrm{He}$ is a member in the association for computing machinery, the association for the natural language processing of Japan. 